TRANSACTIONS OF THE

AMERICAN MATHEMATICAL SOCIETY

Volume 359, Number 7, July 2007, Pages 3357-3367

S 0002-9947(07)04135-9

Article electronically published on January 26, 2007

\title{
TORSION FREENESS OF SYMMETRIC POWERS OF IDEALS
}

\author{
ALEXANDRE B. TCHERNEV
}

\begin{abstract}
Let $I$ be an ideal in a Noetherian commutative ring $R$ with unit, let $k \geq 2$ be an integer, and let $\alpha_{k}: S_{k} I \longrightarrow I^{k}$ be the canonical surjective $R$-module homomorphism from the $k$ th symmetric power of $I$ to the $k$ th power of $I$. When $\operatorname{pd}_{R} I \leq 1$ or when $I$ is a perfect Gorenstein ideal of grade 3, we provide a necessary and sufficient condition for $\alpha_{k}$ to be an isomorphism in terms of upper bounds for the minimal number of generators of the localisations of $I$. When $I=\mathfrak{m}$ is a maximal ideal of $R$ we show that $\alpha_{k}$ is an isomorphism if and only if $R_{\mathfrak{m}}$ is a regular local ring. In all three cases for $I$ our results yield that if $\alpha_{k}$ is an isomorphism, then $\alpha_{t}$ is also an isomorphism for each $1 \leq t \leq k$.
\end{abstract}

\section{INTRODUCTION}

Let $I$ be an ideal in a Noetherian commutative ring $R$ with unit. The symmetric algebra $\mathrm{S}_{R}(I)$, the Rees algebra $R(I)$, and the canonical surjective homomorphism of graded $R$-algebras

$$
\alpha: \mathrm{S}_{R}(I) \longrightarrow R(I)
$$

are central objects of study in both commutative algebra and algebraic geometry; see e.g. [13] and the references cited there. A main question of interest is, given an integer $k \geq 2$, to determine conditions under which the degree $k$ component

$$
\alpha_{k}: \mathrm{S}_{k} I \longrightarrow I^{k}
$$

of the map $\alpha$ is an isomorphism. When $I$ contains a regular element (for example when $\operatorname{pd}_{R} I<\infty$, which will be true in the cases we consider), the assumption that $\alpha_{k}$ is an isomorphism is equivalent to the torsion freeness of $\mathrm{S}_{k} I$. Such an assumption imposes strong restrictions on the ideal $I$, and our goal is to investigate exactly what these restrictions are. Our study was also motivated by the desire to answer the question whether $\alpha_{k}$ being an isomorphism implies that $\alpha_{t}$ is an isomorphism for each $1 \leq t \leq k$. That these questions are difficult to answer in general is evident even when $k=2$. In that case the kernel of $\alpha_{2}$ has a characterization in terms of André-Quillen homology [10], and the ideal $I$ is called syzygetic if $\alpha_{2}$ is an isomorphism. Syzygetic ideals have been studied by many authors (see e.g. [7, 8, 9, 11]), and within several classes of ideals there are nontrivial necessary and sufficient conditions available for $I$ to be syzygetic. For example, when $I=\mathfrak{m}$ is a maximal ideal then $\mathfrak{m}$ is syzygetic if and only if $R_{\mathfrak{m}}$ is a regular local ring [13, and when $\operatorname{pd}_{R} I=1$ and height $I=2$ then $I$ is syzygetic if and only if $I$ is generically a complete intersection 11. It turns out that for these two classes of

Received by the editors October 29, 2004 and, in revised form, July 5, 2005.

2000 Mathematics Subject Classification. Primary 13C12, 13D30, 13A30.

Key words and phrases. Torsion freeness, symmetric algebra, Rees algebra, symmetric powers.

(C)2007 American Mathematical Society 3357

Reverts to public domain 28 years from publication 
ideals, and also for the class of perfect Gorenstein ideals of grade 3, there is enough information available on the structure of the powers of $I$ that, quite surprisingly, allows us to give for any $k \geq 2$ a complete answer to the question of when $\alpha_{k}$ is an isomorphism. The main results of this paper are as follows.

In Theorem 5.1 we consider ideals $I$ of projective dimension at most one, and we provide a necessary and sufficient condition for $\alpha_{k}$ to be an isomorphism in terms of upper bounds on the minimal number of generators of the localisations of $I$.

In Theorem 5.3 we consider perfect Gorenstein ideals $I$ of grade 3, and provide a similar necessary and sufficient condition for $\alpha_{k}$ to be an isomorphism.

In both theorems the necessary and sufficient conditions allow us to conclude that if $\alpha_{k}$ is an isomorphism, then $\alpha_{t}$ is also an isomorphism for $1 \leq t \leq k$, and to describe in the local case explicit minimal free resolutions for the ideals $I^{t}$. The main idea in both proofs is to construct functorially a complex of finite free modules whose homology exhibits rigidity properties analogous to the rigidity properties of the Koszul complex. The construction of this complex is such that $\alpha_{k}$ is an isomorphism precisely when the first homology of the complex vanishes, forcing it to be acyclic and thereby providing the desired necessary and sufficient conditions.

Finally, in our third main result, Theorem [5.5] we consider the case when $I=\mathfrak{m}$ is a maximal ideal. We show that $\alpha_{k}$ is an isomorphism if and only if $R_{\mathfrak{m}}$ is a regular local ring. In particular, $\alpha_{k}$ being an isomorphism implies that $\mathfrak{m}$ has finite projective dimension and that $\alpha_{t}$ is an isomorphism for every $t \geq 0$. We note that at this point we are not aware of an example of an ideal $I$ such that $\alpha_{k}$ is an isomorphism but $\alpha_{k-1}$ is not, and that this certainly remains a very intriguing open question. We conclude this Introduction with some remarks on notation.

Throughout this paper rings are commutative Noetherian with unit, modules are finitely generated and unitary, and complexes are nonnegative (i.e. if $\mathbf{M}$ is a complex, then $M_{i}=0$ for $i<0$ ). An $R$-module is considered as a complex concentrated in homological degree 0 . For a complex $\mathbf{M}$ of $R$-modules we say that $\mathbf{M}$ is acyclic if $\mathrm{H}_{i}(\mathbf{M})=0$ for $i \geq 1$. We say that $\mathbf{M}$ is rigid if for any $R$-module $M$ and any integer $i \geq 0$ the condition $\mathrm{H}_{i}\left(\mathbf{M} \otimes_{R} M\right)=0$ implies $\mathrm{H}_{j}\left(\mathbf{M} \otimes_{R} M\right)=0$ for all $j \geq i$. The quintessential example of a rigid complex is the Koszul complex on a sequence of elements in $R$. Given a ring homomorphism $\rho: R \longrightarrow S$ and a complex of $R$-modules $\mathbf{M}$ we write $S \otimes_{\rho} \mathbf{M}$ for the tensor product $S \otimes_{R} \mathbf{M}$, where we consider $S$ as an $R$-module via the map $\rho$. For a free $R$-module $F$ we set $F^{*}=\operatorname{Hom}_{R}(F, R)$ and identify the free $S$-modules $S \otimes_{\rho} F^{*}$ and $\left(S \otimes_{\rho} F\right)^{*}$ via the standard canonical isomorphism over $S$ that sends $s \otimes \phi$ to the map given by the formula $t \otimes f \longmapsto s t \phi(f)$.

\section{CATEgories AND Generic OBJECTS}

In this section we establish the categorical framework that provides an appropriate setting for the canonical creation of complexes whose homology exhibits good rigidity properties.

1.1. Categories. We write Comp for the category whose objects are the pairs of the form $\{R, \mathbf{F}\}$, where $R$ is a ring and $\mathbf{F}$ is a complex of free $R$-modules. For objects $\{R, \mathbf{F}\}$ and $\{S, \mathbf{G}\}$, the set of morphisms

$$
\operatorname{Mor}_{\mathbf{C o m p}}(\{R, \mathbf{F}\},\{S, \mathbf{G}\})
$$


in Comp is the set of all pairs $\{\rho, \phi\}$, where $\rho: R \longrightarrow S$ is a ring homomorphism and $\phi: S \otimes_{\rho} \mathbf{F} \longrightarrow \mathbf{G}$ is a morphism of complexes over $S$. In particular, given a ring map $\rho: R \longrightarrow S$ and a complex of free $R$-modules $\mathbf{F}$, we have the base change morphism $\rho_{*}:\{R, \mathbf{F}\} \longrightarrow\left\{S, S \otimes_{\rho} \mathbf{F}\right\}$ given by the formula

$$
\rho_{*}=\left\{\rho, \operatorname{id}_{S \otimes_{\rho} \mathbf{F}}\right\} .
$$

For morphisms $\{\rho, \phi\}:\{R, \mathbf{F}\} \longrightarrow\{S, \mathbf{G}\}$ and $\{\sigma, \psi\}:\{S, \mathbf{G}\} \longrightarrow\{T, \mathbf{H}\}$ in Comp their composition is given by the formula

$$
\{\sigma, \psi\} \circ\{\rho, \phi\}=\left\{\sigma \circ \rho, \psi \circ\left(\mathrm{id}_{T} \otimes \phi\right)\right\} .
$$

Note that for each ring $R$ the category $\operatorname{Comp}(R)$ of complexes of free $R$-modules is a subcategory of Comp. (A complex of free $R$-modules $\mathbf{F}$ is identified with the pair $\{R, \mathbf{F}\}$, and a morphism of complexes $\psi$ is identified with the morphism $\left\{\operatorname{id}_{R}, \psi\right\}$.) Furthermore, for a ring map $\rho: R \longrightarrow S$ the base change morphisms $\rho_{*}$ in Comp induce in the obvious way the usual base change functor

$$
\rho_{*}(-): \operatorname{Comp}(R) \longrightarrow \operatorname{Comp}(S) .
$$

Similarly, for an integer $n \geq 1$ we consider the category Comp $^{\times n}$ with objects the $(n+1)$-tuples

$$
\left\{R, \mathbf{F}_{1}, \ldots, \mathbf{F}_{n}\right\},
$$

where each $\mathbf{F}_{i}$ is a complex of free $R$-modules. The morphisms of $\mathbf{C o m p}{ }^{\times n}$ are defined in complete analogy to the way morphisms of Comp were defined. In particular, $\mathbf{C o m p}^{\times 1}$ is nothing but Comp. The categories $\operatorname{Comp}^{\times n}(R)$ and their base change functors $\rho_{*}$ are also defined analogously.

1.2. Closed subcategories. A category $\mathcal{X}$ is called a closed subcategory if it is a subcategory of Comp ${ }^{\times n}$ for some $n \geq 1$ and is closed under base change, i.e. for each object $\left\{R, \mathbf{F}_{1}, \ldots, \mathbf{F}_{n}\right\}$ in $\mathcal{X}$ and each ring homomorphism $\rho: R \longrightarrow S$, the object

$$
\rho_{*}\left(\left\{R, \mathbf{F}_{1}, \ldots, \mathbf{F}_{n}\right\}\right)=\left\{S, S \otimes_{\rho} \mathbf{F}_{1}, \ldots, S \otimes_{\rho} \mathbf{F}_{n}\right\}
$$

is also an object in $\mathcal{X}$, and similarly for morphisms of $\mathcal{X}$. When an object of $\mathcal{X}$ is also an object of $\operatorname{Comp}^{\times n}(R)$, we call it an $R$-object of $\mathcal{X}$. We write $\mathcal{X}(R)$ for the subcategory of the category $\operatorname{Comp}^{\times n}(R)$ with objects the $R$-objects of $\mathcal{X}$ and morphisms those morphisms of $\mathcal{X}$ that are also morphisms of $\operatorname{Comp}^{\times n}(R)$.

The following closed subcategories will be our main examples of interest.

Example 1.2.1. Given integers $s$ and $t$ we write $\mathcal{M}_{s, t}$ for the full subcategory of the category Comp with objects the pairs $\{R, \mathbf{F}\}$ such that $\operatorname{rank} F_{0}=s, \operatorname{rank} F_{1}=t$, and $F_{i}=0$ for $i \geq 2$. Since $\mathcal{M}_{s, t}(R)$ is equivalent (via the obvious identification) to the category of $R$-module maps from free $R$-modules of rank $t$ to free $R$-modules of rank $s$, in the sequel we will abuse terminology and refer to the objects of $\mathcal{M}_{s, t}(R)$ as homomorphisms of $R$-modules.

Example 1.2.2. The closed subcategory $\mathcal{A}_{g}$ of $\mathbf{C o m p}{ }^{\times 2}$ is defined as follows. The $R$-objects of $\mathcal{A}_{g}$ are the triples $\{R, G, \mathbf{G}\}$, where $G$ is a finite free $R$-module of rank $g$ (considered as a complex concentrated in homological degree 0 ) and $\mathbf{G}$ is a complex over $R$ of the form

$$
\mathbf{G}=0 \longrightarrow G^{*} \stackrel{\xi}{\longrightarrow} G \longrightarrow 0
$$

with $G$ as its component in homological degree 0 and such that the map $\xi$ is alternating in the sense that $\phi[\xi(\phi)]=0$ for every $\phi \in G^{*}$. Thus the objects of 
$\mathcal{A}_{g}$ are simply alternating maps in disguise. Furthermore, let $H$ be another free $R$-module of rank $g$, let

$$
\mathbf{H}=0 \longrightarrow H^{*} \stackrel{\chi}{\longrightarrow} H \longrightarrow 0
$$

with $\chi$ an alternating map, and let $\phi: G \longrightarrow H$ be an isomorphism of $R$-modules such that the diagram

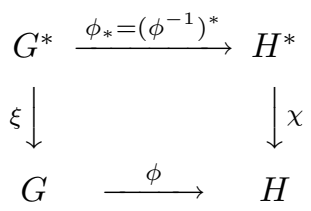

is commutative. In that setting we write

$$
\phi_{\bullet}: \mathbf{G} \longrightarrow \mathbf{H}
$$

for the corresponding induced isomorphism of complexes of $R$-modules. With this notation we can describe the set of morphisms in $\mathcal{A}_{g}$ from an object $\{Q, F, \mathbf{F}\}$ to an object $\{R, H, \mathbf{H}\}$ as the set of all triples of the form

$$
\left\{\rho, \phi, \phi_{\bullet}\right\}
$$

where $\rho: Q \longrightarrow R$ is a ring homomorphism, $G=R \otimes_{\rho} F$, and $\phi: G \longrightarrow H$ is an isomorphism of $R$-modules such that for the complex $\mathbf{G}=R \otimes_{\rho} \mathbf{F}$ the diagram (1.2.5) is commutative.

1.3. Generic objects. Let $\mathbb{k}$ be a ring, and let $R$ be a polynomial ring over $\mathbb{k}$ in finitely many variables. Let $\mathcal{X}$ be a closed subcategory, and let $A$ be an $R$-object in $\mathcal{X}$. We say that $A$ is an $\mathcal{X}$-generic object over $\mathbb{k}$ if for any commutative $\mathbb{k}$-algebra $S$ and any $S$-object $B$ of $\mathcal{X}$ there exists a homomorphism of $\mathbb{k}$-algebras $\rho: R \longrightarrow S$ such that the objects $\rho_{*}(A)$ and $B$ are isomorphic in the category $\mathcal{X}(S)$.

We say that the closed subcategory $\mathcal{X}$ is sufficiently generic if for every ring $\mathbb{k}$ there exists an $\mathcal{X}$-generic object over $\mathbb{k}$.

Remarks 1.3.1. Let $\mathbb{k}$ be a ring.

(a) Let $t$ and $s$ be positive integers. Let $R=\mathbb{k}\left[x_{i j} \mid 1 \leq i \leq s, 1 \leq j \leq t\right]$ be the polynomial ring over $\mathbb{k}$ on the indicated set of variables. Let $F=R^{t}$ and $G=R^{s}$, and let

$$
\mathbf{F}=0 \longrightarrow F \stackrel{\psi}{\longrightarrow} G \longrightarrow 0
$$

be the complex with $G$ in homological degree 0 and such that the matrix for $\psi$ in the standard bases of $F$ and $G$ is $X=\left(x_{i j}\right)$. It is clear that $\{R, \mathbf{F}\}$ is an $\mathcal{M}_{s, t}$-generic over $\mathbb{k}$ object, hence $\mathcal{M}_{s, t}$ is a sufficiently generic closed subcategory.

(b) Let $g \geq 3$ be an odd integer. Let $R=\mathbb{k}\left[x_{i j} \mid 1 \leq i<j \leq g\right]$ be a polynomial ring over $\mathbb{k}$ on the indicated set of variables. Let $G=R^{g}$, and let $\xi: G^{*} \longrightarrow G$ be the alternating map that, in the standard basis of $G$ and the dual basis of $G^{*}$, has matrix $\left(\xi_{i j}\right)$ given by

$$
\xi_{i j}= \begin{cases}x_{i j} & \text { if } i<j \\ 0 & \text { if } i=j \\ -x_{j i} & \text { if } i>j\end{cases}
$$

Let $\mathbf{G}=0 \longrightarrow G^{*} \stackrel{\xi}{\longrightarrow} G \longrightarrow 0$ be the complex with $G$ in homological degree 0 . It is clear that $\{R, G, \mathbf{G}\}$ is an $\mathcal{A}_{g}$-generic over $\mathbb{k}$ object, hence $\mathcal{A}_{g}$ is a sufficiently generic closed subcategory. 


\section{LAYERED FUNCTORS AND RIGIDITY}

Here we introduce the notion of a rigid functor. Rigid functors provide a canonical mechanism for producing complexes whose homology has good rigidity behavior. In the only result of this section, Proposition 2.1, we adapt to our setting a wellknown and very useful criterion for verifying that a functor is rigid. Throughout the section $\mathcal{X}$ is a closed subcategory, and

$$
\mathcal{F}: \mathcal{X} \longrightarrow \text { Comp }
$$

is a functor that preserves $R$-objects for every ring $R$, i.e. we have

$$
\mathcal{F}\left(\left\{R, \mathbf{F}_{1}, \ldots, \mathbf{F}_{n}\right\}\right)=\left\{R, \mathcal{F}_{R}\left(\mathbf{F}_{1}, \ldots, \mathbf{F}_{n}\right)\right\},
$$

where $\mathcal{F}_{R}\left(\mathbf{F}_{1}, \ldots, \mathbf{F}_{n}\right)$ is a complex of finite free $R$-modules, and such that

$$
\mathcal{F}\left(\left\{\rho, \phi_{1}, \ldots, \phi_{n}\right\}\right)=\left\{\rho, \mathcal{F}_{\rho}\left(\phi_{1}, \ldots, \phi_{n}\right)\right\}
$$

for every morphism $\left\{\rho, \phi_{1}, \ldots, \phi_{n}\right\}:\left\{R, \mathbf{F}_{1}, \ldots, \mathbf{F}_{n}\right\} \longrightarrow\left\{S, \mathbf{H}_{1}, \ldots, \mathbf{H}_{n}\right\}$ in $\mathcal{X}$. We write $\mathcal{F}_{R}\left(\phi_{1}, \ldots, \phi_{n}\right)$ for the morphism $\mathcal{F}_{\mathrm{id}_{R}}\left(\phi_{1}, \ldots, \phi_{n}\right)$. Note that this induces for every ring $R$ a functor

$$
\mathcal{F}_{R}: \mathcal{X}(R) \longrightarrow \operatorname{Comp}(R) .
$$

We will call the functor $\mathcal{F}$ a layered functor if in addition for every ring homomorphism $\rho: R \longrightarrow S$ there is an isomorphism of functors

$$
\beta_{\rho}:\left(\mathcal{F}_{S} \circ \rho_{*}\right) \longrightarrow\left(\rho_{*} \circ \mathcal{F}_{R}\right) .
$$

We say that $\mathcal{F}$ is a rigid functor if it is a layered functor, and in addition for each $i \geq 0$ and for each object $\left\{S, \mathbf{H}_{1}, \ldots, \mathbf{H}_{n}\right\}$ in $\mathcal{X}$ one has $\mathrm{H}_{i}\left(\mathcal{F}_{S}\left(\mathbf{H}_{1}, \ldots, \mathbf{H}_{n}\right)\right)=0$ if and only if $\mathrm{H}_{j}\left(\mathcal{F}_{S}\left(\mathbf{H}_{1}, \ldots, \mathbf{H}_{n}\right)\right)=0$ for all $j \geq i$.

The following proposition is well-known to experts, however, since there seems to be no reference in the literature that covers the generality we require, we present a proof.

Proposition 2.1. Let $\mathcal{X}$ be a sufficiently generic closed subcategory, let

$$
\mathcal{F}: \mathcal{X} \longrightarrow \text { Comp }
$$

be a layered functor, and assume that for every ring $\mathbb{k}$ there exists an $\mathcal{X}$-generic over $\mathbb{k}$ object $\left\{R, \mathbf{F}_{1}, \ldots, \mathbf{F}_{n}\right\}$ such that the complex $\mathcal{F}_{R}\left(\mathbf{F}_{1}, \ldots, \mathbf{F}_{n}\right)$ is acyclic. Then the functor $\mathcal{F}$ is rigid.

Proof. Let $S$ be a ring, let $\left\{S, \mathbf{H}_{1}, \ldots, \mathbf{H}_{n}\right\}$ be an $S$-object of $\mathcal{X}$, and let $\mathbf{G}=$ $\mathcal{F}_{S}\left(\mathbf{H}_{1}, \ldots, \mathbf{H}_{n}\right)$. Let $\left\{R, \mathbf{F}_{1}, \ldots, \mathbf{F}_{n}\right\}$ be an $\mathcal{X}$-generic over $S$ object such that the complex $\mathcal{F}_{R}\left(\mathbf{F}_{1}, \ldots, \mathbf{F}_{n}\right)$ is acyclic. Thus there exists an $S$-algebra map $\rho: R \longrightarrow S$ such that there is an isomorphism in $\mathcal{X}(S)$

$$
\left\{S, S \otimes_{\rho} \mathbf{F}_{1}, \ldots, S \otimes_{\rho} \mathbf{F}_{n}\right\} \cong\left\{S, \mathbf{H}_{1}, \ldots, \mathbf{H}_{n}\right\},
$$

hence we have an isomorphism of complexes of $S$-modules

$$
\mathcal{F}_{S}\left(S \otimes_{\rho} \mathbf{F}_{1}, \ldots, S \otimes_{\rho} \mathbf{F}_{n}\right) \cong \mathcal{F}_{S}\left(\mathbf{H}_{1}, \ldots, \mathbf{H}_{n}\right),
$$

and therefore the isomorphism $\beta_{\rho}\left(\left\{R, \mathbf{F}_{1}, \ldots, \mathbf{F}_{n}\right\}\right)$ induces an isomorphism of the complex $S \otimes_{\rho} \mathcal{F}_{R}\left(\mathbf{F}_{1}, \ldots, \mathbf{F}_{n}\right)$ with the complex $\mathbf{G}$. Since $R$ is a polynomial ring over $S$ and the map $\rho$ is an $S$-algebra map, $\rho$ is surjective and induces an isomorphism of $R$-modules $S \cong R / I$, where $I$ is an ideal in $R$ generated by an $R$-regular 
sequence $x_{1}, \ldots, x_{m}$. Also, let $M$ be the zeroth homology of the acyclic complex $\mathcal{F}_{R}\left(\mathbf{F}_{1}, \ldots, \mathbf{F}_{n}\right)$. Then

$$
\mathrm{H}_{i}(\mathbf{G}) \cong \mathrm{H}_{i}\left(S \otimes_{\rho} \mathcal{F}_{R}\left(\mathbf{F}_{1}, \ldots, \mathbf{F}_{n}\right)\right)=\operatorname{Tor}_{i}^{R}(S, M)=\mathrm{H}_{i}\left(\mathbf{K} \otimes_{R} M\right),
$$

where $\mathbf{K}$ is the Koszul complex over $R$ on the sequence $x_{1}, \ldots, x_{m}$. Since the Koszul complex is rigid the proposition follows.

\section{Graded strands of the Koszul complex}

The goal of this section is to define for each integer $q \geq 0$ the layered functors

$$
\mathcal{K}^{q}: \mathcal{M}_{s, t} \longrightarrow \text { Comp and } \widetilde{\mathcal{K}}^{q}: \mathcal{M}_{t+1, t} \longrightarrow \text { Comp, }
$$

and to show in Theorem 3.1 and Theorem 3.3 that $\mathcal{K}^{q}$ and $\widetilde{\mathcal{K}}^{q}$ are rigid functors. These results will provide in Section 5 the key ingredient in the proof of the first main result of this paper, Theorem 5.1. We proceed first with the definition of $\mathcal{K}^{q}$.

Let $F$ and $G$ be free $R$-modules of ranks $t$ and $s$, respectively, with $t \leq s$. Let $\psi: F \longrightarrow G$ be a homomorphism, and let

$$
\mathbf{F}=0 \longrightarrow F \stackrel{\psi}{\longrightarrow} G \longrightarrow 0
$$

be the corresponding complex of $R$-modules with $G$ in (homological) degree 0 . Let $e_{1}, \ldots, e_{t}$ be a basis of the module $F$, let

$$
S=\bigoplus_{i \geq 0} \mathrm{~S}_{i} G
$$

be the symmetric algebra of $G$ over $R$, and write $\mathcal{K}(\mathbf{F})$ for the Koszul complex over $S$ on the elements $\psi\left(e_{1}\right), \ldots, \psi\left(e_{t}\right)$. The complex $\mathcal{K}(\mathbf{F})$ splits into a direct sum of complexes of free $R$-modules

$$
\mathcal{K}(\mathbf{F})=\bigoplus_{q \geq 0} \mathcal{K}_{R}^{q}(\mathbf{F})
$$

(see e.g. [5, §A2.6.1]), where the $q$ th graded strand $\mathcal{K}_{R}^{q}(\mathbf{F})$ has the form

$$
\mathcal{K}_{R}^{q}(\mathbf{F})=0 \longrightarrow F_{n} \longrightarrow F_{n-1} \longrightarrow \ldots \longrightarrow F_{1} \longrightarrow F_{0} \longrightarrow 0,
$$

with $F_{i}=\bigwedge^{i} F \otimes_{R} \mathrm{~S}_{q-i} G$ and $n=\min \{q, t\}$. It is clear from the definition that if $\phi: \mathbf{F} \longrightarrow \mathbf{G}$ is a morphism of complexes over $R$, it induces canonically a morphism of complexes $\mathcal{K}_{R}^{q}(\phi): \mathcal{K}_{R}^{q}(\mathbf{F}) \longrightarrow \mathcal{K}_{R}^{q}(\mathbf{G})$. Since for every ring map $\rho: R \longrightarrow S$ we have by construction a canonical isomorphism $S \otimes_{\rho} \mathcal{K}_{R}^{q}(\mathbf{F}) \cong \mathcal{K}_{S}^{q}\left(S \otimes_{\rho} \mathbf{F}\right)$, it is easy to check that the assignment $\{R, \mathbf{F}\} \longrightarrow\left\{R, \mathcal{K}_{R}^{q}(\mathbf{F})\right\}$ induces a layered functor

$$
\mathcal{K}^{q}: \mathcal{M}_{s, t} \longrightarrow \text { Comp }
$$

Theorem 3.1. The functor $\mathcal{K}^{q}$ is rigid for each $q \geq 0$.

We need the following acyclicity criterion of Avramov [1, Proof of Proposition 1].

Theorem $3.2([1])$. The complex $\mathcal{K}_{R}^{q}(\mathbf{F})$ is acyclic if and only if grade $I_{t-i+1}(\psi) \geq$ $i$ for each $1 \leq i \leq \min (q, t)$. In particular, $\mathcal{K}_{R}^{q}(\mathbf{F})$ is acyclic for the $\mathcal{M}_{s, t}$-generic over $\mathbb{k}$ object $\{R, \mathbf{F}\}$ from Remarks 1.3.1(a).

Proof of Theorem 3.1. As the category $\mathcal{M}_{s, t}$ is sufficiently generic, by Proposition 2.1 it is enough show that for every ring $\mathbb{k}$ the complex $\mathcal{K}_{R}^{q}(\mathbf{F})$ is acyclic, where $\{R, \mathbf{F}\}$ is the $\mathcal{M}_{s, t}$-generic over $\mathbb{k}$ object from Remarks 1.3.1(a). This however is immediate from Theorem 3.2 , 
For the rest of this section we restrict ourselves to the special case when $s=t+1$, and proceed with the definition of the functor $\widetilde{\mathcal{K}}^{q}$. We have a homomorphism

$$
\epsilon: G \longrightarrow \widetilde{G}
$$

(with $\widetilde{G}=\bigwedge^{t+1} G \otimes_{R} \bigwedge^{t} F^{*}$ ) defined as the composition

$$
G \stackrel{\iota}{\longrightarrow} \bigwedge^{t+1} G \otimes \bigwedge^{t} G^{*} \stackrel{1 \otimes \bigwedge \psi^{*}}{\longrightarrow} \bigwedge^{t+1} G \otimes \bigwedge^{t} F^{*}
$$

where $\iota: G \longrightarrow\left(\bigwedge^{t+1} G^{*} \otimes \bigwedge^{t} G\right)^{*}$ is the canonical isomorphism induced by the exterior multiplication pairing

$$
\bigwedge^{t+1} G^{*} \otimes \bigwedge^{t} G \otimes G \stackrel{1 \otimes \wedge}{\longrightarrow} \bigwedge^{t+1} G^{*} \otimes \bigwedge^{t+1} G \stackrel{\mu}{\longrightarrow} R .
$$

Note that the free $R$-module $\widetilde{G}$ is isomorphic to $R$, and the cokernel of $\epsilon$ is an $R$ module isomorphic to $R / I$, where $I=I_{t}(\psi)$ is the ideal of $t \times t$ minors of the map $\psi$. Furthermore, a standard exercise in multilinear algebra shows that $\epsilon \circ \psi=0$, therefore $\epsilon$ factors through the cokernel $M$ of $\psi$. It follows that when $q \geq 0$ the map

$$
\epsilon_{q}: \mathrm{S}_{q} G \stackrel{S_{q} \epsilon}{\longrightarrow} \mathrm{S}_{q} \widetilde{G}
$$

has cokernel isomorphic to $R / I^{q}$, and factors through $\mathrm{S}_{q} M=\mathrm{H}_{0}\left(\mathcal{K}_{R}^{q}(\mathbf{F})\right)$. Therefore for each $q \geq 0$ the map $\epsilon_{q}$ augments the complex $\mathcal{K}_{R}^{q}(\mathbf{F})$ to obtain a complex

$$
\widetilde{\mathcal{K}}_{R}^{q}(\mathbf{F})=0 \longrightarrow F_{n} \longrightarrow F_{n-1} \longrightarrow \ldots \longrightarrow F_{1} \longrightarrow F_{0} \stackrel{\epsilon_{q}}{\longrightarrow} \mathrm{S}_{q} \widetilde{G} \longrightarrow 0
$$

shifted so that $\mathrm{S}_{q} \widetilde{G}$ is the component in homological degree 0 . It is clear from the definitions that the assignment $\{R, \mathbf{F}\} \longmapsto\left\{R, \widetilde{\mathcal{K}}_{R}^{q}(\mathbf{F})\right\}$ induces a layered functor

$$
\widetilde{\mathcal{K}}^{q}: \mathcal{M}_{t+1, t} \longrightarrow \text { Comp } .
$$

Theorem 3.3. The functor $\widetilde{\mathcal{K}}^{q}$ is rigid.

Proof. By Proposition 2.1 it suffices to show that for every ring $\mathbb{k}$ the complex $\widetilde{\mathcal{K}}_{R}^{q}(\mathbf{F})$ is acyclic, where $\{R, \mathbf{F}\}$ is the $\mathcal{M}_{t+1, t}$-generic over $\mathbb{k}$ object from Remarks 1.3.1(a). Recall that $R$ is a polynomial ring over $\mathbb{k}$, and for some choice of bases for the modules $F$ and $G$ the entries of the matrix $X$ of $\psi$ are the variables over $\mathbb{k}$. Therefore the ideal $I=I_{t}(\psi)$ is perfect of grade 2 . Also it is well known, see e.g. [3, (2.5)], that for each $1 \leq i \leq t$ we have

$$
\text { grade } I_{t-i+1}(\psi)=i(i+1) \geq i+1 \text {. }
$$

Therefore by Theorem 3.2 the complex $\mathcal{K}_{R}^{q}(\mathbf{F})$ is acyclic and $\mathrm{H}_{0}\left(\mathcal{K}_{R}^{q}(\mathbf{F})\right)=\mathrm{S}_{q} I$ is torsion free, hence isomorphic to $I^{q}$. Since $\epsilon_{q}$ factors through $\mathrm{H}_{0}\left(\mathcal{K}_{R}^{q}(\mathbf{F})\right) \cong I^{q}$ and has as its image $I^{q}\left(\mathrm{~S}_{q} \widetilde{G}\right) \cong I^{q}$, the desired acyclicity of the complex $\widetilde{\mathcal{K}}_{R}^{q}(\mathbf{F})$ follows.

\section{Complexes Associated With AN Alternating MAP}

The goal of this section is to define for each odd integer $g \geq 3$ and each integer $q \geq 1$ the layered functors

$$
\mathcal{D}^{q}: \mathcal{A}_{g} \longrightarrow \text { Comp } \quad \text { and } \quad \widetilde{\mathcal{D}}^{q}: \mathcal{A}_{g} \longrightarrow \text { Comp }
$$

and to show in Theorem 4.3 that they are rigid functors. These results will provide in Section 5 the key ingredient in the proof of the second main result of this paper, Theorem 5.3. We proceed first with the definition of $\mathcal{D}^{q}$. 
Let $g \geq 3$ be an odd integer, let $\{R, G, \mathbf{G}\}$ be an object in $\mathcal{A}_{g}$ with

$$
\mathbf{G}=0 \longrightarrow G^{*} \stackrel{\xi}{\longrightarrow} G \longrightarrow 0
$$

and let $M$ be the cokernel of $\xi$. For any integer $q \geq 1$ Kustin and Ulrich $[6$ and Boffi and Sánchez 2] construct in a canonical way a finite complex $\mathcal{D}^{q}(\xi)=\mathcal{D}_{R}^{q}(G, \mathbf{G})$ of free modules over $R$ of the form

$$
\mathcal{D}_{R}^{q}(G, \mathbf{G})=0 \longrightarrow G_{\mu} \longrightarrow \ldots \longrightarrow G_{0} \longrightarrow 0
$$

with $G_{0}=\mathrm{S}_{q} G$ and $\mathrm{H}_{0}\left(\mathcal{D}^{q}(G, \mathbf{G})\right)=\mathrm{S}_{q} M$. For details on the construction and properties of the complexes $\mathcal{D}_{R}^{q}(G, \mathbf{G})$ we refer to [6] and [2], and also to [12, where a concise summary is given. As the presentation in [12, Section 1] appears to be best suited to our purpose, for the rest of this section we follow the notation introduced there.

In particular, when $\left\{\operatorname{id}_{R}, \phi, \phi_{\bullet}\right\}$ is a morphism in $\mathcal{A}_{g}(R)$ from $\{R, G, \mathbf{G}\}$ to $\{R, H, \mathbf{H}\}$ with $\mathbf{H}$ as in (1.2.4), then it is easy to check from the definition of the maps $\xi^{(k)}$ and $\chi^{(k)}$ that the diagram

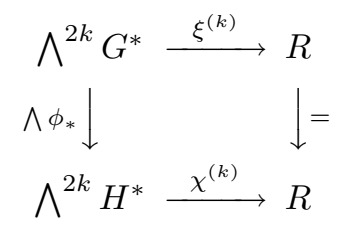

commutes. It follows for the maps $d_{\xi}^{(q)}$ and $d_{\chi}^{(q)}$ that when $q=g-2 k$ the diagram

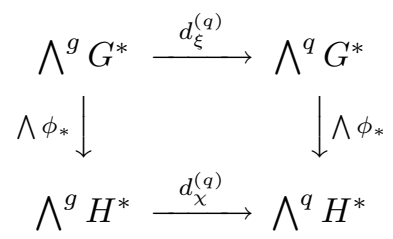

is commutative. On the other hand, the homomorphism $\phi \otimes \phi_{*}: G \otimes G^{*} \longrightarrow H \otimes H^{*}$ sends the trace element $\eta_{\xi}$ to the trace element $\eta_{\chi}$, hence we have an induced isomorphism of DG-algebras over $R$

$$
\overline{\mathbb{K}}\left(\phi_{\bullet}\right): \overline{\mathbb{K}}(\xi) \longrightarrow \overline{\mathbb{K}}(\chi) .
$$

Therefore for each $q \geq 1$ we have an induced morphism of complexes of free $R$ modules

$$
\mathcal{D}_{R}^{q}\left(\phi, \phi_{\bullet}\right): \mathcal{D}_{R}^{q}(G, \mathbf{G}) \longrightarrow \mathcal{D}_{R}^{q}(H, \mathbf{H}) .
$$

It is also clear from the construction of the complex $\mathcal{D}_{R}^{q}(G, \mathbf{G})$ that for every ring homomorphism $\rho: R \longrightarrow S$ there is a canonical isomorphism of complexes of free $S$-modules

$$
\beta_{\rho}: S \otimes_{\rho} \mathcal{D}_{R}^{q}(G, \mathbf{G}) \longrightarrow \mathcal{D}_{S}^{q}\left(S \otimes_{\rho} G, S \otimes_{\rho} \mathbf{G}\right) .
$$

It follows that the assignment $\{R, G, \mathbf{G}\} \longrightarrow\left\{R, \mathcal{D}_{R}^{q}(G, \mathbf{G})\right\}$ induces for each $q \geq 1$ a layered functor

$$
\mathcal{D}^{q}: \mathcal{A}_{g} \longrightarrow \text { Comp }
$$

Next, we proceed with the definition of the functor $\widetilde{\mathcal{D}}^{q}$. We set $\widehat{G}=\bigwedge^{g} G^{*}$. Thus the free $R$-module $\widehat{G}$ is isomorphic to $R$, and we consider the map

$$
\delta: G \stackrel{\left(d_{\xi}^{(1)}\right)^{*}}{\longrightarrow} \widehat{G} .
$$


It is clear from the definitions that $\operatorname{Im}(\delta)=I \widehat{G}$, where $I=\operatorname{Pf}_{g-1}(\xi)$ is the ideal of submaximal Pfaffians of $\xi$. Since $\xi^{*}=-\xi$ and $\mathcal{D}_{R}^{1}(G, \mathbf{G})$ is a complex, it follows that $\delta \circ \xi=0$ and so $\delta$ factors through the module $M=\operatorname{Coker}(\xi)$. Therefore

$$
\delta_{q}: \mathrm{S}_{q} G \stackrel{\mathrm{S}_{q} \delta}{\longrightarrow} \mathrm{S}_{q} \widehat{G}
$$

has image $I^{q} \mathrm{~S}_{q} \widehat{G} \cong I^{q}$ and augments $\mathcal{D}_{R}^{q}(G, \mathbf{G})$ to the complex

$$
\widetilde{\mathcal{D}}_{R}^{q}(G, \mathbf{G})=0 \longrightarrow G_{\mu} \longrightarrow \ldots \longrightarrow G_{0} \stackrel{\delta_{q}}{\longrightarrow} \mathrm{S}_{q} \widehat{G} \longrightarrow 0,
$$

shifted so that $\mathrm{S}_{q} \widehat{G}$ is the component in homological degree 0 . It is now straightforward to verify that the assignment $\{R, G, \mathbf{G}\} \longrightarrow\left\{R, \widetilde{\mathcal{D}}_{R}^{q}(G, \mathbf{G})\right.$ induces a layered functor

$$
\widetilde{\mathcal{D}}^{q}: \mathcal{A}_{g} \longrightarrow \text { Comp }
$$

The following theorem is the main result of this section.

Theorem 4.3. The layered functors $\mathcal{D}^{q}$ and $\widetilde{\mathcal{D}}^{q}$ are rigid.

Proof. Since $\mathcal{A}_{g}$ is a sufficiently generic closed subcategory, by Proposition 2.1 it suffices to show that for every ring $\mathbb{k}$ there is an $\mathcal{A}_{g}$-generic over $\mathbb{k}$ object $\{R, G, \mathbf{G}\}$ such that the complexes $\mathcal{D}_{R}^{q}(G, \mathbf{G})$ and $\widetilde{\mathcal{D}}_{R}^{q}(G, \mathbf{G})$ are acyclic.

Let $\{R, G, \mathbf{G}\}$ be the $\mathcal{A}_{g}$-generic over $\mathbb{k}$ object from Remarks 1.3.1(b). Then by [6. Theorem 7.3] and [2] the complex $\mathcal{D}_{R}^{q}(G, \mathbf{G})$ is acyclic and $\mathrm{H}_{0}\left(\mathcal{D}_{R}^{q}(G, \mathbf{G})\right) \cong I^{q}$. Thus the augmentation map $\delta_{q}$ induces a surjection of $I^{q}$ onto itself, which must be an isomorphism. The acyclicity of the complex $\widetilde{\mathcal{D}}_{R}^{q}(G, \mathbf{G})$ is now immediate.

\section{TORSION FREENESS}

Here we present the statements and the proofs of the three main results of this paper. Throughout the section for an ideal $J$ we set $V(J)=\{\mathfrak{p} \in \operatorname{Spec}(R) \mid J \subseteq \mathfrak{p}\}$, and we write $\mu(J)$ for the minimal number of generators of $J$.

Theorem 5.1. Let $I$ be an ideal in a Noetherian ring $R$ with $\operatorname{pd}_{R} I \leq 1$, and let $k \geq 2$ be an integer. Set $m=\sup \left\{\mu\left(I_{\mathfrak{p}}\right) \mid \mathfrak{p} \in V(I)\right\}$. The following conditions are equivalent:

(1) $\mathrm{S}_{k} I \cong I^{k}$;

(2) $\mathrm{S}_{t} I \cong I^{t}$ for every $1 \leq t \leq k$;

(3) $\mu\left(I_{\mathfrak{p}}\right) \leq \operatorname{depth} R_{\mathfrak{p}}$ for every $\mathfrak{p} \in V(I)$ such that depth $R_{\mathfrak{p}} \leq \min \{m, k\}$.

Remarks 5.2. (a) The special case $k=2$ of Theorem 5.1 is due to Simis and Vasconcelos [11, Theorem 2.2].

(b) If $R$ is local and $\mathbf{F}=0 \longrightarrow F \longrightarrow G \longrightarrow 0$ is the minimal free resolution of $I$, then by Theorem 3.2 condition (3) is equivalent to the complex $\mathcal{K}_{R}^{k}(\mathbf{F})$ being a minimal free resolution of the ideal $I^{k}$, and is therefore also equivalent to the complex $\mathcal{K}_{R}^{t}(\mathbf{F})$ being a minimal free resolution of the ideal $I^{t}$ for each $1 \leq t \leq k$.

Proof of Theorem 5.1. It is clear that we may assume $R$ to be local with maximal ideal $\mathfrak{m}$, and that $\mu(I)=m$. Then (3) implies (2) by Remarks 5.2 (b) and (2) implies (1) trivially, thus we proceed to show that (1) implies (3).

Note that by the Hilbert-Burch theorem the ideal $I$ has a minimal free resolution of the form

$$
\mathbf{F}=0 \longrightarrow F \stackrel{\psi}{\longrightarrow} G \longrightarrow 0,
$$


where $F$ is of rank $m-1$, the free $R$-module $G$ has rank $m$, and grade $I_{m-1}(\psi)=2$. Consider the complex $\widetilde{\mathcal{K}}_{R}^{k}(\mathbf{F})$. The condition $\mathrm{S}_{k} I \cong I^{k}$ means that $\mathrm{H}_{1}\left(\widetilde{\mathcal{K}}_{R}^{k}(\mathbf{F})\right)=0$. Since $\widetilde{\mathcal{K}}^{k}$ is a rigid functor by Theorem 3.3 , this condition is equivalent to the acyclicity of $\widetilde{\mathcal{K}}_{R}^{k}(\mathbf{F})$. By construction this is if and only if the complex $\mathcal{K}^{k}(\mathbf{F})$ is a free resolution of $I^{k}$, which by Remarks 5.2 (b) is equivalent to the inequalities from condition (3).

Theorem 5.3. Let I be a perfect Gorenstein ideal of grade 3 in a Noetherian ring $R$, and let $k \geq 2$ be an integer. Let $m$ be as in the statement of Theorem [5.1, and let $p=m-1-2 \max \left\{0,\left\lfloor\frac{m-k-1}{2}\right\rfloor\right\}$. The following conditions are equivalent:

(1) $\mathrm{S}_{k} I \cong I^{k}$;

(2) $\mathrm{S}_{t} I \cong I^{t}$ for every $1 \leq t \leq k$;

(3) $\mu\left(I_{\mathfrak{p}}\right) \leq \operatorname{depth} R_{\mathfrak{p}}$ for each $\mathfrak{p} \in V(I)$ with $\operatorname{depth} R_{\mathfrak{p}} \leq p$.

Remark 5.4. We note that if $R$ is local, then according to the structure theorem of Buchsbaum and Eisenbud [4 the ideal $I$ has a minimal free presentation by an alternating map $\xi: G^{*} \longrightarrow G$ with $\mu(I)=\operatorname{rank} G=m \geq 3$ odd, and we let

$$
\mathbf{G}=0 \longrightarrow G^{*} \stackrel{\xi}{\longrightarrow} G \longrightarrow 0
$$

be the corresponding complex. In that situation, by [6, Theorem 6.2] and [12, Theorem B], condition (3) is equivalent to the complex $\mathcal{D}_{R}^{k}(G, \mathbf{G})$ being a minimal free resolution of the ideal $I^{k}$, and is therefore also equivalent to the complex $\mathcal{D}_{R}^{t}(G, \mathbf{G})$ being a minimal free resolution of the ideal $I^{t}$ for each $1 \leq t \leq k$.

Proof of Theorem 5.3. It is clear we may assume that $R$ is a local ring, and that we have $G, \xi$, and $\mathbf{G}$ as in Remark [5.4. Then (3) implies (2) by Remark 5.4 and (2) implies (1) trivially, thus we proceed with the proof that (1) implies (3).

Since the functor $\widetilde{\mathcal{D}}^{k}$ is rigid by Theorem 3.3 , and since condition (1) is equivalent to $\mathrm{H}_{1}\left(\widetilde{\mathcal{D}}_{R}^{k}(G, \mathbf{G})\right)=0$, it follows that (1) implies the acyclicity of $\widetilde{\mathcal{D}}_{R}^{k}(G, \mathbf{G})$. Therefore $\mathcal{D}_{R}^{k}(G, \mathbf{G})$ is a resolution of $I^{k}$, and the desired conclusion is now immediate from Remark [5.4.

Theorem 5.5. Let $\mathfrak{m}$ be a maximal ideal in a Noetherian ring $R$, and let $k \geq 2$ be an integer. The following conditions are equivalent:

(1) $\mathrm{S}_{k} \mathfrak{m} \cong \mathfrak{m}^{k}$;

(2) $\mathrm{S}_{t} \mathfrak{m} \cong \mathfrak{m}^{t}$ for every $t \geq 1$;

(3) the ring $R_{\mathfrak{m}}$ is a regular local ring.

Remark 5.6. The case $k=2$ of Theorem [5.5 is the content of [13, Corollary 2.1.4]. In particular, conditions (2) and (3) of Theorem 5.5] are already known to be equivalent.

Proof of Theorem 5.5. It is clear that we may assume the $\operatorname{ring} R$ is local with maximal ideal $\mathfrak{m}$. Then the implications $(3) \Longrightarrow(2) \Longrightarrow(1)$ are clear. Furthermore by Remark 5.6 we have $(2) \Longrightarrow(3)$, thus it remains to show that $(1) \Longrightarrow(2)$. We prove this by induction on $k$. The cases $k=1$ and $k=2$ are trivial, so we assume that $k \geq 3$ and that (1) implies (2) for $k-1$. To complete the proof it is enough to show that if $\alpha_{k}$ is an isomorpism, then also $\alpha_{k-1}$ is an isomorpism. Let

$$
\lambda_{k}: \mathrm{S}_{k} \mathfrak{m} \longrightarrow \mathrm{S}_{k-1} \mathfrak{m}
$$


be the $R$-module homomorphism defined by the formula $y_{1} \ldots y_{k} \longrightarrow y_{1} \cdot\left(y_{2} \ldots y_{k}\right)$. Suppose $K \neq 0$ is the kernel of $\alpha_{k-1}$. Since the image of $\lambda_{k}$ is $\mathfrak{m} \mathrm{S}_{k-1} \mathfrak{m}$, it is immediate that $\alpha_{k}=\alpha_{k-1} \circ \lambda_{k}$. Therefore $K \cap \mathfrak{m} \mathrm{S}_{k-1} \mathfrak{m}=0$, hence $K$ is a nonzero vector space over $R / \mathfrak{m}$ consisting of minimal generators of $\mathrm{S}_{k-1} \mathfrak{m}$. Let $x \in K$ with $x \neq 0$. Then for each minimal generator $y$ of $\mathfrak{m}$ the element $z=y x$ is nonzero in $\left(\mathrm{S}_{k} \mathfrak{m}\right) / \mathfrak{m}\left(\mathrm{S}_{k} \mathfrak{m}\right)=\mathrm{S}_{k}\left(\mathfrak{m} / \mathfrak{m}^{2}\right)$, hence is a nonzero minimal generator of $\mathrm{S}_{k} \mathfrak{m}$ with

$$
\alpha_{k}(z)=\alpha_{k-1}\left(\lambda_{k}(y x)\right)=\alpha_{k-1}(y \cdot x)=y \alpha_{k-1}(x)=0,
$$

a contradiction. Thus $\alpha_{k-1}$ is injective, hence an isomorphism.

\section{REFERENCES}

[1] L. Avramov, Complete intersections and symmetric algebras, J. Algebra 73 (1981), 248-263. MR0641643 (83e:13024)

[2] G. Boffi and R. Sánchez, On the resolutions of the powers of the Pfaffian ideal, J. Algebra 152 (1992), 463-491. MR1194315 (93j:14065)

[3] W. Bruns and U. Vetter, Determinantal Rings, Lecture Notes in Math. 1327, SpringerVerlag, Berlin 1988. MR0953963 (89i:13001)

[4] D. Buchsbaum and D. Eisenbud, Algebraic structures for finite free resolutions and some structure theorems for ideals of codimension 3, Amer. J. Math. 99 (1977), 447-485. MR $0453723(56: 11983)$

[5] D. Eisenbud, Commutative algebra with a view toward algebraic geometry, Graduate Texts in Math. 150, Springer-Verlag, New York 1995. MR.1322960 (97a:13001)

[6] A. Kustin and B. Ulrich, A family of complexes associated to an almost alternating map, with applications to residual intersections, Mem. Amer. Math. Soc. 95 (1992), no. 461. MR.1091668(92i:13012)

[7] A. Micali and N. Roby, Algèbres symétriques et syzygies, J. Algebra 17 (1971), 460-469. MR 0282964 (44:198)

[8] A. Micali, P. Salmon, and P. Samuel, Integrité et factorialité des algèbres symétriques, Atas do IV Coloquio Brasileiro de Matematica, Sao Paolo 1965. MR.0207741 (34:7556)

[9] F. Planas-Vilanova, Rings of weak dimension one and syzygetic ideals, Proc. Amer. Math. Soc. 124 (1996), no. 10, 3015-3017. MR1328371 (96m:13005)

[10] T. Porter, Homology of commutative algebras and an invariant of Simis and Vasconcelos, J. Algebra 99 (1986), 458-465. MR0837555 (87i:13006)

[11] A. Simis and W. Vasconcelos, The syzygies of the conormal module, Amer. J. Math. 103 (1981), 203-224. MR0610474 (82i:13016)

[12] A. Tchernev, Acyclicity criteria for complexes associated with an alternating map, Proc. Amer. Math. Soc. 129 (2001), no. 10, 2861-2869. MR.1840088 (2002d:13017)

[13] W. Vasconcelos, Arithmetic of blowup algebras, Cambridge University Press, Cambridge 1994. MR 1275840 (95g:13005)

Department of Mathematics, University at Albany, SUny, Albany, New York 12222

E-mail address: tchernev@math.albany.edu 